\title{
Modelo sistemático para utilização dos recursos e ferramentas da Plataforma Moodle: uma proposta para mediação da aprendizagem no Ensino Superior
}

\author{
Natana Lopes Pereira \\ natanapereiralopes@hotmail.com - PPGTIC/UFSC \\ Prof. Dr. Fernando José Spanhol \\ profspanhol@gmail.com - PPGTIC/UFSC \\ Prof. Dr. Giovani Mendonça Lunardi \\ giovaniunir@gmai.com - PPGTIC/UFSC
}

\section{Resumo}

Devido à necessidade da utilização eficiente e eficaz dos recursos tecnológicos disponibilizados nos Ambientes Virtuais de Ensino e Aprendizagem - AVEA, o artigo apresenta um roteiro para construção e conversão de conhecimento em tais plataformas educacionais. Assim, a pesquisa objetiva a partir de um modelo inicial, proporcionar maior efetividade nos AVEA, por meio da aplicação dos quatro modos de conversão do conhecimento. Para delimitação da pesquisa, realizou-se estudo bibliográfico sobre os modos de conversão do conhecimento e a plataforma educacional Moodle. A partir da elaboração e estruturação do roteiro para conversão do conhecimento, evidenciou-se a relevância da combinação dos recursos de forma sistemática, para o processo de ensino e aprendizagem mediados por tais ferramentas.

Palavras-chave: Construção do Conhecimento; Modelo SECI; AVEA; Tecnologias da Informação e Comunicação; Mediação. 


\section{Systematic model for the use of the resources and tools of the Moodle Platform: a proposal for mediation of learning in Higher Education}

\section{Abstract}

Due to the need for efficient and effective use of the technological resources available in the Virtual Environments of Teaching and Learning, the article presents a roadmap for building and converting knowledge in such educational platforms. Thus, the research aims from an initial model, to provide greater effectiveness in AVEA through the application of the four modes of knowledge conversion. For the delimitation of the research, a bibliographic study on the ways of knowledge conversion and the educational platform Moodle. From the elaboration and structuring of the roadmap for knowledge conversion, the relevance of the combination of resources in a systematic way for the teaching and learning process mediated by such tools was evidenced.

Keywords: Construction of Knowledge; SECI Model; Virtual Environments of Teaching Learning; Informationand Communication Technologies; Mediation.

\section{Modelo sistemático para utilización de los recursos y hierramientas de la plataforma moodle: una propuesta para mediación del aprendizaje en la Enseñanza Superior}

\section{Resumen}

Debido a la necesidad del uso eficiente y eficaz de los recursos tecnológicos disponibles en los Ambientes Virtuales de Enseñanza y Aprendizaje AVEA, el artículo presenta un itinerario para construcción y conversión de conocimiento en tales plataformas educativas. Así, la investigación objetiva a partir de un modelo inicial, proporcionar mayor efectividad en los AVEA por medio de la aplicación de los cuatro modos de conversión del conocimiento. Para delimitación de la investigación se realizó un estudio bibliográfico sobre los modos de conversión del conocimiento y la plataforma educativa Moodle. A partir de la elaboración y estructuración del itinerario para la conversión del conocimiento, se evidenció la relevancia de la combinación de los recursos de forma sistemática para el proceso de enseñanza y aprendizaje mediados por tales herramientas. 
Palabras clave: Construcción del conocimiento; Modelo SECI; Ambientes Virtuales de enseñanza y aprendizaje; Tecnologías de la Información y Comunicación; Mediación.

\section{Introdução}

No atual contexto acadêmico, são constantes as inovações tecnológicas que visam atender à nova forma de construção de conhecimento, desencadeada devido à integração das Tecnologias da Informação e Comunicação - TIC - em práticas diárias. Nesse viés, uma das principais ferramentas que vêm sendo gradativamente aderidas no contexto acadêmico pelas instituições de ensino, como potenciais tecnologias para construção do conhecimento, são os Ambientes Virtuais de Ensino e Aprendizagem - AVEA. Tais plataformas possuem diversos recursos e ferramentas de TIC, que incentivam a construção do conhecimento tanto de forma individual (interação aluno-objeto), quanto colaborativa (interação em grupo), possibilitando, e contribuindo para mediação do processo de ensino e aprendizagem (HOSSEINI, 2011).

Estudos de Dahlstrom, Brookse e Bichsel (2014) evidenciam que alunos e professores consideram tais plataformas relevantes para a realização de práticas acadêmicas, mas que utilizam apenas suas funcionalidades básicas de disponibilização de conteúdo didático, e envio de mensagens. Corroborando com tal informação, Seixas et al. (2012) destacam o uso primário dos AVEA apenas como mero repositório de informações, não empregando seus recursos de TIC como potenciais ferramentas para a construção do conhecimento. Nesse viés, Alarcon (2015) identifica a necessidade de elaborar novas estratégias no ensino superior, por meio do estudo quanto ao planejamento do método aplicado, e em como utilizar tais recursos para tornar efetivo o processo de ensino e aprendizagem.

Assim, evidencia-se a necessidade de combinar áreas distintas do conhecimento, rompendo barreiras epistemológicas, por meio de pesquisas interdisciplinares, para propor inovações, e/ou novas formas de construção de conhecimento, no processo de ensino e aprendizagem de nível superior (CARDOSO et al., 2008). A partir 
do cenário exposto, e da necessidade de aprimorar o uso das TIC integradas no contexto educacional evidenciados por Alarcon (2015), identificou-se o objetivo desta pesquisa em propor um roteiro para utilização de ferramentas e recursos da plataforma Moodle de forma eficiente e eficaz, visando auxiliar na mediação da construção do conhecimento.

Para tal, combinaram-se estudos bibliográficos e exploratórios sobre os modos de conversão do conhecimento (SECI) de Tackeuchi e Nonaka (2008), e sobre os Ambientes Virtuais de Ensino e Aprendizagem, descritos na seção dois (2) desta pesquisa. No item três (3), uma breve explicitação dos métodos utilizados para realização do presente estudo. $\mathrm{Na}$ seção quatro, como resultado da pesquisa, além da classificação dos recursos e ferramentas da plataforma Moodle, com base no modelo SECI, apresenta-se a sequência didática elaborada para uso efetivo das tecnologias dos AVEA.

\section{Fundamentação teórica}

\subsection{Gestão do Conhecimento - A abordagem do modelo SECI aplicada no Ensino Superior}

Segundo Spiegler (2002 apud LACERDA et al., 2010), o conhecimento reside na mente do ser humano, podendo o mesmo ser capturado, armazenado e compartilhado, sendo uma difícil tarefa sua mensuração. Criar conhecimento não é apenas a capacidade de aprender com o outro, mas também a capacidade de construir conhecimento por si só, e por meio da interação com outros membros (LACERDA et al.,2010). Nesse viés, Davenport e Prusak (1998) enfatizam que os termos conhecimento, informação e dados não são sinônimos, sendo de extrema importância, para melhor entendimento do processo de construção do conhecimento, sua distinção. Os dados são registros estruturados de transações, que podem se transformar em informação. Por sua vez, a informação, pode dar forma aos dados. Quando trabalhada na mente humana, de modo a realizar comparação, analisar a consequência de tal 
informação, suas conexões com o conhecimento já acumulado, pode gerar o conhecimento (DAVENPORT; PRUSAK, 1998).

Assim, o conhecimento segundo Nonaka e Takeuchi (1997), significa sabedoria adquirida, por meio da interação. Além da relação de aprendizagem aluno e objeto, em uma visão epistemológica, Uriarte (2008 apud PACHECO, 2015) elenca que a construção do conhecimento ocorre por intermédio da interação entre duas formas de conhecimentos: explícito e tácito. O conhecimento explícito é externalizado por meio da linguagem formal, da escrita, de manuais, documentos, ou outras formas de codificação, já o conhecimento tácito é mais difícil de transmitir, uma vez que é resultante da incorporação de experiências individuais, que envolve fatores intangíveis como crenças pessoais, perspectivas, entre outros (NONAKA; TAKEUCHI, 1997).

Nesse contexto, a partir da possibilidade de interação entre o conhecimento tácito e explícito Takeuchi e Nonaka (2008), elaboraram o modelo de conversão do conhecimento SECI. De acordo com Takeuchi e Nonaka (2008), o modelo SECI, foi inicialmente utilizado para promover o intercâmbio de conhecimento entre os profissionais de uma organização e, atualmente, sendo aderido ao contexto no ensino superior, para melhoria e inovação quanto ao método de ensino (THONGKOO; PANJABUREE; DAUGCHARONE, 2017).

Tal modelo também conhecido como espiral do conhecimento, é composto por quatro modos de conversão do conhecimento - Socialização, Externalização, Combinação e Internalização -, resultantes da sua interação de forma cíclica e contínua (TAKEUCHI; NONAKA, 2008). Segundo Vaccaro, Veloso e Brusoni (2009), é uma das poucas abordagens que se concentram na relação (interação) de forma cíclica entre diferentes tipos de conhecimentos (tácito e explícito). 
Figura 1: Modelo SECI.

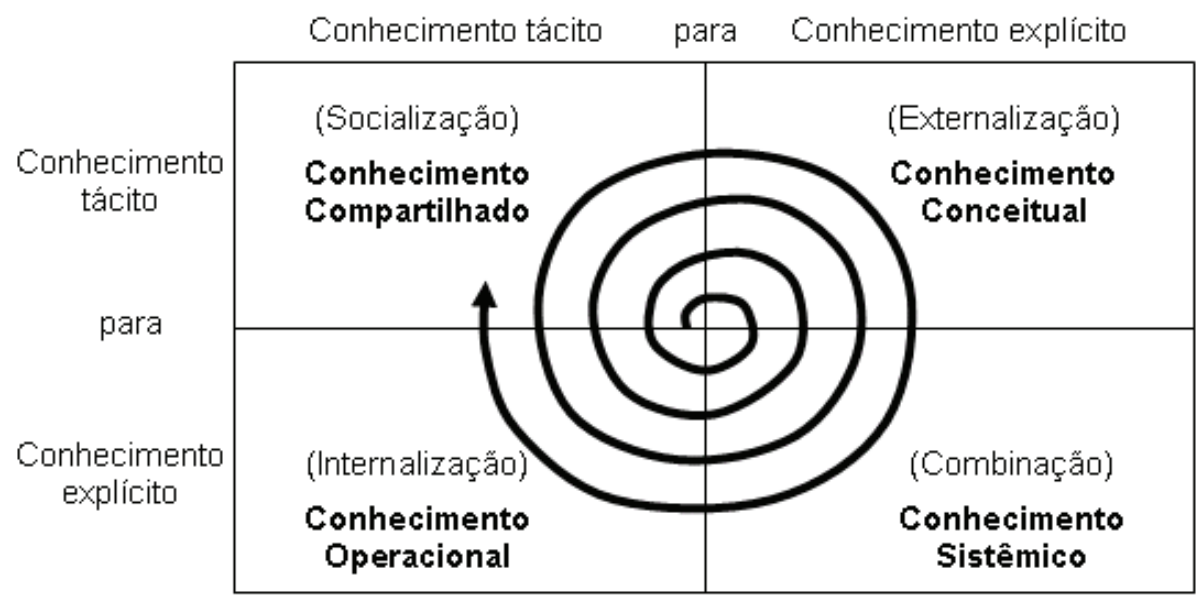

Fonte: Takeuchi e Nonaka (2008).

Nesse ciclo, para conversão do conhecimento, primeiramente ocorre a Socialização, sendo caracterizada pelo compartilhamento de conhecimento (experiências) - do tácito para tácito (TAKEUCHI; NONAKA, 2008). Assim, o conhecimento tácito se converte em mais conhecimento tácito. Em seguida, após a troca de experiências e vivências, Takeuchi e Nonaka (2008) enfatizam a necessidade de externalizar, codificar o conhecimento tácito, por meio da escrita, fala, imagens, vídeos, entre outros, caracterizando o quadrante de Externalização. Tal modo (externalização), de acordo com Takeuchi e Nonaka (2008), referese a tradução do conhecimento tácito em formas compreensivas para outra pessoa. Na Combinação, ocorre a colocação do conhecimento recém-criado e do já existente proveniente de outras seções. Resulta assim na "combinação" do conhecimento recém-explicitado com o conhecimento explícito já existente (explícito/ explícito). Por fim, a Internalização, é decorrente da incorporação do conhecimento explícito em tácito. Tal conversão consiste no aprender fazendo, em outras palavras, é a construção de um novo conhecimento na prática (TAKEUCHI; NONAKA, 2008).

No contexto educacional, algumas ações de gestão de conhecimento, como o modelo SECI, vêm atualmente sendo utilizadas por meio de ambientes colaborativos, tendo como principal objetivo a construção e o compartilhamento 
do conhecimento. Os processos para criação e compartilhamento de conhecimento, ainda não se tornaram práticas diárias em rotinas acadêmicas, sendo primordial o incentivo dessas ações no ensino superior, para proporcionar ambientes colaborativos, interativos e dinâmicos (BIASUTTI; EL-DEGHAIDY, 2012).

Segundo Humboldt (2011 apud BIASUT'TI; ELDEGHAIDY, 2012), o principal objetivo do ensino superior é a criação de conhecimento utilizando a pesquisa acadêmica; disseminação do conhecimento por intermédio da educação; e serviços acadêmicos prestados à sociedade, sendo a transferência de conhecimento para a sociedade. Esses itens demonstram o relacionamento, e a importância da Gestão do Conhecimento - GC no ensino superior, sendo relevante o uso das TIC para potencializar o processo de ensino e aprendizagem, por meio de métodos de conversão do conhecimento.

Tais modos de conversão do conhecimento aplicados no ensino superior podem ser potencializados pelas TIC. As mídias sociais digitais, oferecem ótimas oportunidades para construção de espaços de interação, entre o conhecimento tácito de uma pessoa para outra (socialização). As ferramentas wiki, fóruns, blog, entre outros recursos de comunicação, possibilitam a externalização de conhecimentos tácitos, sendo de extrema relevância o uso de tais recursos. Essas tecnologias, além da externalização do conhecimento, possibilitam sua combinação com outros conhecimentos tácitos externalizados pelos discentes, sendo também uma forma de aprendizagem colaborativa. Para a internalização do conhecimento, ressaltam-se as ferramentas de simulação, jogos, e atividades que incentivem o discente a construção do conhecimento por meio de atividades práticas (HOSSEINI, 2010).

\subsection{Recursos e Ferramentas de Ambientes Virtuais de Ensino e Aprendizagem}

As Novas Tecnologias da Informação e Comunicação NTIC - proporcionaram o desenvolvimento e aperfeiçoamento 
de AVEA. Esses "ambientes" facilitam o aprendizado, fazendo que o processo ocorra de forma "amigável", além de instigar o aluno a construir seu conhecimento, de acordo com sua forma de aprendizagem, sem a necessidade de estar presencialmente em uma sala de aula (CAPUTI; GARRIDO, 2015).

Segundo Gonçalves e Bernardi (2012), o AVEA proporciona um novo paradigma no processo de ensinar e aprender. O processo educacional, anteriormente ao advento tecnológico, era condicionado a uma sala de aula composta por carteiras e um quadro-negro, não possibilitando qualquer forma de inovação na aprendizagem, por meio de recursos de Tecnologias da Informação e Comunicação - TIC. Atualmente, com o desenvolvimento de plataformas educacionais, surgem novas possibilidades de ensino e aprendizagem, tornando o aluno, cada vez mais independente nesse processo.

Nesse contexto, Torres (2007 apud GONÇALVES; BERNARDI, 2012) enfatizam a importância dos recursos de TIC nas plataformas educacionais, citando como exemplo o Modular Objetc Oriented Dynamic Learning Environmet - Moodle, que atualmente é um dos AVEA mais utilizados do mundo. De acordo com Gonçalves e Bernardi (2012), ele é um software livre em constante evolução, que permite a comunicação, informação e o ensino e aprendizagem por meio de seus diversos recursos de TIC. Tais tecnologias disponibilizadas pelas plataformas virtuais são classificas em dois grupos por Silva (2011): (i) recursos, e (ii) ferramentas.

Os recursos, segundo Silva (2011), possibilitam a disponibilização de conteúdo didático de modo virtual em diversas mídias, ou a indicação de fontes de conteúdos por meio de links ou páginas web. Já as ferramentas, tornam possível a elaboração de atividades de forma colaborativa ou individual, visando auxiliar na construção do conhecimento. Contribuem também no processo de mediação do ensino e aprendizagem, além de possibilitar a realização de pesquisas de avaliação da própria disciplina, entre outras funcionalidades. De acordo com Lacerda (2013 apud 
PEREIRA; MENDES; SPANHOL, 2017), por meio de suas diversas ferramentas, os AVEA possibilitam uma nova forma de aprendizagem, conforme o novo perfil do aluno. É de grande importância o domínio da tecnologia utilizada, do conteúdo a ser abordado e principalmente da forma, ou método de aplicação no ambiente (PEREIRA; MENDES; SPANHOL, 2017).

\section{Organização do método de estudo}

Para realização do presente estudo, primeiramente efetuaram-se buscas exploratórias, visando à identificação na literatura de trabalhos que comportassem os termos considerados relevantes para a pesquisa, sendo eles: "AVEA", "Educação Superior", "Modelo SECI". A partir dos resultados obtidos, pôdese constatar a aderência a práticas de Gestão do Conhecimento GC no ensino superior, todavia evidenciou-se defasagem quanto a trabalhos interdisciplinares integrando os quadrantes de conversão de conhecimento (SECI), ao contexto acadêmico.

De acordo com a hipótese criada neste estudo, referente à contribuição do modelo SECI para conversão e criação do conhecimento por meio de AVEA e mediação de tal processo, além da pesquisa bibliográfica, realizou-se análise qualitativa dos artigos relacionados. Essa abordagem visou analisar a interação entre as variáveis envolvidas no estudo (FREIRE, 2013), sendo selecionados os artigos considerados de maior relevância.

Após a identificação dos trabalhos, iniciou-se o processo de planejamento da disciplina, definição dos recursos e ferramentas do Moodle, e elaboração da sequência didática, com vistas a tornar mais eficiente o processo de ensino e aprendizagem, por meio dos AVEA, conforme descrito na Figura 2: 
Figura 2: Etapas da pesquisa.
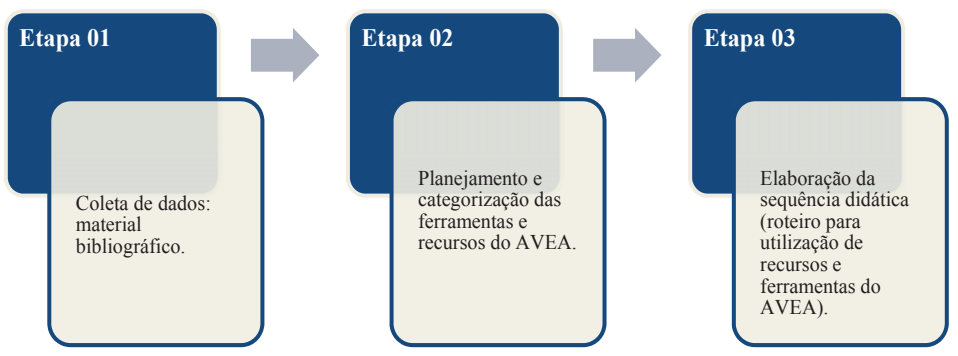

Fonte: Desenvolvido pelo(s) autor(es).

\section{Apresentação e discussão dos resultados}

\subsection{Abordagem Modelo SECI - Ferramentas e Recursos do Moodle}

Após o planejamento da pesquisa, foram identificados na busca exploratória da literatura (CAPUTI; GARRIDO, 2015; BIASUTTI; EL-DEGHAIDY, 2012; AHMAD et al., 2012; APARCI, 2017; HOSSEINI, 2011; THONGKOO; PANJABUREE; DAUGCHARONE, 2017) recursos e ferramentas de TIC de AVEA aplicáveis a um conjunto de atividades, de acordo com modos de conversão do conhecimento de Takeuchi e Nonaka (2008) - Modelo SECI. Segundo Hosseini (2011), tal modelo pode ser aplicado ao cenário acadêmico, por meio da aplicação de um conjunto sistêmico de tarefas e rotinas para a construção do conhecimento, utilizando como principal espaço para conversão o AVEA.

A partir dessa concepção inicial, e para a elaboração de rotinas didáticas, inicialmente classificaram-se os recursos e ferramentas da plataforma Moodle, conforme os modos de conversão do conhecimento (SECI). Cabe destacar, que podem ocorrer mudanças na categorização das TIC listadas no Quadro 1, uma vez que, de acordo com o objetivo do docente, alguns recursos e atividades podem ser utilizados tanto de forma individual, quanto coletiva. As TIC evidenciadas são as mais usadas de acordo com Silva (2011), mas 
enfatiza-se que a plataforma Moodle está em constante atualização, e disponibiliza diversos plugins, ${ }^{1}$ com várias aplicabilidades.

Quadro 1: Análise das ferramentas e recursos do Moodle e os modos de conversão.

\begin{tabular}{|l|l|l|l|l|l|}
\hline TIC - MOODLE & CLASSIFICAÇÃO & S & E & C & I \\
\hline Fórum & Ferramenta assíncrona - Colaborativa & $\mathrm{x}$ & $\mathrm{x}$ & $\mathrm{x}$ & \\
\hline Chat & Ferramenta síncrona - Colaborativa & $\mathrm{x}$ & $\mathrm{x}$ & & \\
\hline Wiki & $\begin{array}{l}\text { Ferramenta Colaborativa ou Indivi- } \\
\text { dual }\end{array}$ & $\mathrm{x}$ & & $\mathrm{x}$ & \\
\hline Livro & Recurso Colaborativo ou Individual & & & $\mathrm{x}$ & \\
\hline Diretórios (Pasta) & Recurso colaborativo ou Individual & & & $\mathrm{x}$ & \\
\hline Questionário & Ferramenta Individual & & & $\mathrm{x}$ \\
\hline Lição & Ferramenta Individual & & & $\mathrm{x}$ \\
\hline Tarefa & Ferramenta Individual & & & $\mathrm{x}$ \\
\hline URL (Link a um site) & Recurso & & & $\mathrm{x}$ & \\
\hline Ferramenta Externa & Recurso & & & $\mathrm{x}$ & $\mathrm{x}$ \\
\hline Página & Recurso & & $\mathrm{x}$ & \\
\hline Arquivo & Recurso & & $\mathrm{x}$ & \\
\hline Glossário & Ferramenta Colaborativa & & & $\mathrm{x}$ & \\
\hline
\end{tabular}

Fonte: Desenvolvido pelo(s) autor(es).

Assim, com base na categorização dos recursos e ferramentas realizados para a sequência didática proposta neste artigo, constatouse que tais TIC podem ser aderidas em mais de um modo de conversão do conhecimento. As ferramentas de comunicação, como o fórum e o chat, impulsionam a socialização, modo de conversão em que ocorre o compartilhamento do conhecimento tácito para tácito. Segundo Hosseini (2011), tais recursos contribuem para maior interação aluno-aluno, aluno-conteúdo, aluno-instrutor e aluno e espaço virtual de aprendizagem.

plugin ou módulo de extensão é um programa de computador usado para adicionar funções a outros programas maiores, provendo alguma funcionalidade especial ou muito específica. Fonte:https://pt.wikipedia.org/wiki/Plug-in 
Além de contribuir para a socialização do conhecimento, as ferramentas fórum e chat possibilitam a externalização do conhecimento (do tácito para o explícito). Hosseini (2011) enfatiza que essa é a fase de maior dificuldade de conversão, devido à falta de compreensão do próprio conhecimento já adquirido (tácito), tornando difícil sua externalização, onde o que, de acordo com a experiência de uma pessoa, parece óbvio, para outras não é. Para isso é necessário, segundo Hosseini (2011), que o discente codifique tal conhecimento por meio de mensagens, que serão recebidas e decodificadas pelo grupo.

Além da externalização, a combinação do conhecimento também pode ser potencializada por meio das ferramentas e recursos colaborativos (wiki, fórum, glossário), ou com a utilização dos recursos (livro, diretório, URL, ferramenta externa, página e arquivo). Todas essas TIC ocasionam a agregação de conhecimentos já existentes com novos conhecimentos (explícito e explícito).

A partir desse ciclo, a internalização acontece quando o conhecimento explícito é convertido em tácito, ocorrendo, segundo Thongkoo, Panjaburee e Daugcharone (2017), por meio de atividades que incentivem o aprender fazendo. Hosseini (2011) evidencia que essa fase pode ser demorada, e não muito clara na percepção dos discentes, uma vez que não pode ser evidenciada ou mensurada por intermédio de gráficos ou figuras. Essa etapa torna possível que o aluno compreenda, e possa direcionar novos conhecimentos (HOSSEINI, 2011). Nesse viés, a realização de tais atividades de forma sistêmica, completa o ciclo de conversão do conhecimento. Cabe destacar, que tal método é contínuo, e pode contribuir no processo de ensino e aprendizagem, uma vez que incentiva maior interação entre o aluno e o AVEA.

\subsection{Sequência didática}

Conforme evidenciado por Silva (2011), o AVEA possui diversas TIC que visam contribuir no processo de ensino e aprendizagem. Uma questão que vem preocupando os docentes na utilização de tal tecnologia é a conversão dos diversos, até então, "dados" disponibilizados em "conhecimento". Nesse viés, estudos 
de Ahmad et al. (2012), Hosseini (2011), Thongkoo, Panjaburee, e Daugcharone (2017), enfatizam a aplicabilidade do modelo de Takeuchi e Nonaka (2008) - Modelo SECI em Ambientes Virtuais de Ensino e Aprendizagem - AVEA, para otimizar o processo de construção do conhecimento.

Os autores (AHMAD et al., 2012, HOSSEINI; 2011, THONGKOO; PANJABUREE; DAUGCHARONE, 2017) mensuram, no contexto educacional, a contribuição da aplicação do modelo SECI em AVEA no desenvolvimento cognitivo, na tomada de decisão, entre outros itens abordados. Assim, visando maior interação do acadêmico com o ambiente, elaborou-se uma sequência didática (estrutura do Moodle), de acordo com os quatro quadrantes do modelo SECI - Socialização, Externalização, Combinação e Internalização, conforme ilustrado na Figura 3.

Conforme sequência explicitada, a socialização do conhecimento ocorre em aula. Essa fase é referente à troca de conhecimento tácito entre os atores envolvidos (alunos/alunos, e alunos/docentes). Nessa etapa, há necessidade de maior interação entre os discentes, tanto de forma presencial (em aula) ou virtual, por meio das ferramentas fórum e chat, sendo o docente, um instrutor em tal processo. Ahmad et al.(2012) corroboram com tal informação, enfatizando a importância da mediação pelo instrutor (professor), uma vez que ele considera a socialização um modo de conversão dependente.

Além da socialização em aula, torna-se relevante para o modelo de construção (conversão) do conhecimento, a disponibilização de material didático on-line, em diversas mídias, impulsionando a combinação do conhecimento. Tal funcionalidade proporciona interação com o conteúdo, de acordo com o estilo de aprendizagem do discente. Após a socialização em aula, o modelo elaborado permite a construção de um espaço de interação entre os envolvidos, por meio de ferramentas colaborativas, ocorrendo assim a externalização do conhecimento. As atividades devem influenciar a explicitação dos conhecimentos tácitos dos acadêmicos. Além de explicitar tais conhecimentos, a ferramenta utilizada (fórum) possibilita também a combinação de conhecimento, uma vez que são compartilhados no mesmo ambiente. 
Figura 3:Estrutura do modelo proposto.

\section{Aula 01}

\section{Descriçào:}

- Apresentaçäo da disciplina e do plano de ensino. Ciéncia, pesquisa e conhecimento cientifico.

- Fundamentos teórico-metodológicos.

- Interdisciplinaridade na pesquisa

\section{Socializaçăo em Aula}

Plano Ensino 158 sxb Documento PDF

O que exatamente é um doutorado.pdf 1.1Mb Documento PDF

pseudociencia.pdr 243kb Documento PDF

\section{Atividade Colaborativa}

\section{Glossário Metodologia da Pesquisa Cientilica}

Fonum, Atividade 1 - Discuta os seguintes itens sobre o filme CRLCÇAO -

Um filme sobre Darwin

\section{Atividade Individual}

O que ê interdisciplinariedade

\section{Material Complementar}

Conhecimento Cientilico

Filme Criação

Fonte: moodle.ufsc.br 
No quadrante de internalização do conhecimento (explícito para tácito) sugere-se a utilização das seguintes ferramentas: hot potatoes, enquete, escolha, lição, questionário e tarefa. Tais tecnologias possibilitam que o discente aprenda na prática. Assim, a estrutura aplicada no Moodle visa, através da realização sistêmica e contínua das etapas, maior aproveitamento do conteúdo disponibilizado no ambiente, onde a cada tópico ou temática é gerado novo ciclo aleatoriamente.

Ressalta-se que o modelo ilustrado é uma proposta para futura aplicação, não sendo considerado neste artigo o resultado de sua utilização em aula. Após a implementação da plataforma educacional Moodle, conforme ilustrado na Figura 2, evidenciou-se que tal ambiente digital comporta diversos recursos e ferramentas que possibilitam a conversão e disseminação do conhecimento, por meio principalmente da interação e colaboração entre os atores envolvidos, viabilizando a conversão dos dados em conhecimento.

\section{Considerações finais}

O objetivo desta pesquisa foi propor um roteiro para utilização de ferramentas e recursos da plataforma Moodle, de forma eficiente e eficaz, visando contribuir na mediação do processo de ensino e aprendizagem. A realização de tal estudo foi motivada pela necessidade de aprimorar o uso das TIC integradas no contexto educacional, evidenciada por Alarcon (2015). Para tal, por meio de buscas de forma exploratória e bibliográfica, categorizaram-se as ferramentas da plataforma educacional Moodle, com base nos quatro quadrantes do modelo SECI (Socialização, Externalização, Combinação e Externalização), e elaborou-se uma sequência didática para a utilização dos recursos e ferramentas do AVEA de forma sistêmica.

Por intermédio da análise dos resultados obtidos neste estudo, pôde-se constatar a aplicabilidade do roteiro proposto nas plataformas educacionais, para maior exploração do ambiente, e de seus diversos recursos e ferramentas. Um dos obstáculos encontrado no decorrer 
da investigação foi a escassez de pesquisas interdisciplinares, abordando o modelo SECI no contexto educacional, e seu uso em plataformas virtuais, evidenciando-se assim a relevância do estudo elaborado. Ahmad et al. (2012) identificam em suas investigações, a importância do compartilhamento do conhecimento dos docentes com os discentes por intermédio de novas práticas no processo de ensino e aprendizagem utilizando as TIC.

Estendendo a discussão para trabalhos futuros, além da aplicação da proposta apresentada para validação desta pesquisa, torna-se eminente a realização de novas pesquisas propondo boas práticas para aperfeiçoar o processo de ensino e aprendizagem por meio dos AVEA. Assim, identifica-se a necessidade de maior investigação, por meio de estudos interdisciplinares, relacionando o espaço virtual de aprendizagem (Ba) e a utilização de boas práticas para mediação, interação e colaboração em AVEA.

\section{Agradecimentos}

À Fundação de Amparo à Pesquisa e Inovação do Estado de Santa Catarina - FAPESC e a Coordenação de Aperfeiçoamento de Pessoal de Nível Superior-CAPES, pela bolsa de estudo concedida.

\section{Referências}

AHMAD, Mazida et al. Investigating the Knowledge Creation Processes in a Learning Management System (LMS). Knowledge Management International Conference. Malaysia, p. 652-656, jul. 2012.

ALARCON, Dafne Fonseca. DIRETRIZES PARA PRÁTICAS DE GESTÃO DO CONHECIMENTO NA EDUCAÇÃO A DISTÂNCIA,2015, $2013 \mathrm{~s}$. Tese (Doutorado) - Curso de Programa de Pós-graduação em Engenharia e Gestão do Conhecimento, Departamento de Engenharia e Gestão do Conhecimento, Universidade Federal de Santa Catarina, Florianópolis, 2015.

ARPACI, I. Antecedents and consequences of cloud computing adoption in education to achieve knowledge management. Computers in Human Behavior, v. 70, p. 382-390, 2017.

BIASUTTI, Michele; EL-DEGHAIDY, Heba. Using Wiki in teacher education: 
Impact on knowledge management processes and student satisfaction. Computers \& Education[s.l.], v. 59, n. 3, p.861-872, nov. 2012. Elsevier BV. http://dx.doi. org/10.1016/j.compedu.2012.04.009.

CAPUTI, Valentina; GARRIDO, Antonio. Student-oriented planning of e-learning contents for Moodle. Journal Of Network And Computer Applications[s.l.], v. 53, p.115-127, jul. 2015. Elsevier BV. http://dx.doi. org/10.1016/j.jnca.2015.04.001. Disponível em:<http://www.sciencedirectcom. ez46.periodicos.capes.gov.br/science/article/pii/S108480451500065X>. Acesso em: $1^{\circ}$. mai. 2017.

CARDOSO, F. S. et al.Interdisciplinaridade: fatos a considerar. R. B. E. C. T., v.1, n. 1, jan./abr. 2008.

DAHLSTROM, E., BROOKS, D. C., \& BICHSEL, J. (2014). The current ecosystem of learning management systems in higher education: Student, faculty, and IT perspectives Research report. Louisville, CO: ECAR.

DAVENPORT, T. H.; PRUSAK, L. Conhecimento empresarial. Rio de Janeiro: Campus, 1998.

DIAS, Rosana de Fátima. AMBIENTES VIRTUAIS DE APRENDIZAGEM - UMA METODOLOGIA PARA AVALIAÇÃO DE SOFTWARE,2013, 151s. Dissertação (Mestrado) - Curso de Engenharia de Produção, Universidade Federal de Santa Catarina, Florianópolis, 2003.

FREIRE, Patrícia de Sá. Aumente qualidade e quantidade de suas publicações científicas: Manual para elaboração de projetos e artigos científicos, 1. ed. Curitiba, PR: CRV, 2013.

GONÇALVES, Angélica Ilha; BERNARDI, Giliane. Moodle: ambiente virtual de aprendizagem de espanhol? A visão dos professores. Revista Horizontes de Linguística Aplicada, Santa Maria, v. 11, n. 1, p.81-102, jun. 2012.

HOSSEINI, S. M. The application of SECI model as a framework of knowledge creation in virtual learning. Asia Pacific Education Review, v. 12, n. 2, p. 263 $270,2011$.

LACERDA, Mário Roberto Miranda etal. CRIAÇÃOECOMPARTILHAMENTO DE CONHECIMENTO EM AMBIENTES VIRTUAIS DE ENSINOAPRENDIZAGEM. Renote - Revista Novas Tecnologias na Educação,Porto Alegre, v. 1, n. 8, p.1-10, jul. 2010. Disponível em: <http://seer.ufrgs.br/index. $\mathrm{php} / \mathrm{renote} /$ article/view/15189/8955>. Acesso em: $1^{\circ}$. mar. 2017. 
LISBÔA, Maria da Graça Portela; GODOY, Leoni Pentiado. APLICAÇÃO DO MÉTODO 5W2H NO PROCESSO PRODUTIVO DO PRODUTO: A JOIA. Revista Iberoamericana de Engenharia Industrial, Florianópolis, v. 4, n. 7, p.32-47, 15 fev. 2012.

NONAKA, Ikujiro; TAKEUCHI, Hirotaka. Criação de conhecimento na empresa: Como as Empresas Japonesas Geram a Dinâmica da Inovação. 2. ed. Rio de Janeiro: Campus, 1997. 358 p.PACHECO, Daniela Chagas. MATURIDADE EM GESTÃO DO CONHECIMENTO DA PREFEITURA MUNICIPAL DE CRICIÚMA: O CASO DOS SETORES DE CONVÊNIOS,2015, 190 s. Dissertação (Mestrado) - Curso de Gestão do Conhecimento, Universidade Federal de Santa Catarina, Florianópolis, 2015.

PEREIRA, Natana L.; MENDES, Angelita D.; SPANHOL, Fernando J.ROTEIRO DE ATIVIDADES EM AMBIENTES VIRTUAIS DE APRENDIZAGEMPARAMEDIAÇÃODETRABALHOSDECONCLUSÃODE CURSO. Renote -Novas Tecnologias na Educação, v. 15, n. 1. Disponível em:http://seer.ufrgs.br/index.php/renote/article/view/75161. Acesso em 20 de ago. 2017.

SEIXAS, Carlos Albertoet al. Ambiente virtual de aprendizagem: estruturação de roteiro para curso online. Revista Brasileira de Enfermagem[s.l.], v. 65, n. 4, p.660-666, ago. 2012. FapUNIFESP (SciELO). http://dx.doi.org/10.1590/ s0034-71672012000400016.

SILVA, R. S. Moodle para autores e tutores, 2. ed. São Paulo: Novatec Editora, 2011.

TAKEUCHI, H; NONAKA, I. Gestão do Conhecimento. Porto Alegre: Bookman, 2008.

THONGKOO, K.; PANJABUREE, P.; DAUNGCHARONE, K. An Inquiry Blended SECI Model-Based Learning Support Approach for Promoting Perceptions and Learning Achievement of University Students. In: HASHIMOTO, K.;FUKUTA, N., et al., 2017. Institute of Electrical and Electronics Engineers Inc. p.527-532.

VACCARO, A.; VELOSO, F; BRUSONI, S. The impact of virtual technologies on knowledge-based processes: An empirical study. Research Policy, v. 38, n. 8, p. $1.278-1.287,2009$.

Submetido em: 25-10-2018

Aceito em: 30-11-2018 